\title{
TWO WEIGHT $\Phi$-INEQUALITIES FOR THE HARDY OPERATOR, HARDY-LITTLEWOOD MAXIMAL OPERATOR, AND FRACTIONAL INTEGRALS
}

\author{
LAI QINSHENG
}

(Communicated by J. Marshall Ash)

\begin{abstract}
Suppose $\Phi$ is an appropriate Young's function and $w(x), v(x)$ are nonnegative locally integrable functions. Let $T$ denote one of three linear operators of special importance that map suitable functions on $R^{n}$ into functions on $R^{n}$.
\end{abstract}

For the Hardy operator $T$, we study the inequality

$$
\int_{0}^{\infty} \Phi(|T f(x)|) w(x) d x \leq C \int_{0}^{\infty} \Phi(|f(x)|) v(x) d x
$$

and for the Hardy-Littlewood maximal operator or fractional integrals $T$, we discuss the inequalities

$$
\int_{R^{n}} \Phi(|T(f v)(x)|) w(x) d x \leq C \int_{R^{n}} \Phi(|f(x)|) v(x) d x .
$$

In all cases we obtain the necessary and sufficient conditions.

\section{INTRODUCTION}

We shall be concerned with integral inequalities of the form

$$
\left.\int_{R^{n}} \Phi|T f(x)|\right) d w \leq C \int_{R^{n}} \Phi(|f(x)|) d \mu,
$$

where $d w, d \mu$ are positive Borel measures on $R^{n}, \Phi$ is an even Young's function on $R$ with $\Phi(0)=0$, and $T$ is one of three linear operators of special importance that map suitable functions on $R^{n}$ into functions on $R^{n}$. Apart from their intrinsic interest, such inequalities are important in application, since they imply the boundedness of $T$ as a map between the associated Orlicz spaces. It is of particular interest to obtain estimates for the best constant $C$ in (1.1).

The first of the cases we consider is the Hardy operator

$$
T f(x)=\int_{0}^{x} f(t) d t, \quad x \in R^{+}=(0, \infty) .
$$

Received by the editors September 24, 1990 and, in revised form, August 19, 1991. 1991 Mathematics Subject Classification. Primary 42B25.

Key words and phrases. Young's function, Hardy operator, maximal operator, fractional integral. This work was supported in part by the Universities' China Committee in London. 
Let $\phi$ be a nondecreasing right continuous function on $R^{+}$with $\phi(0+)=0$, and define $\Phi$ to be Young's function $\Phi(t)=\int_{0}^{|t|} \phi(s) d s$. Let $\phi^{-1}$ be the right continuous inverse of $\phi$ and set $\Psi(t)=\int_{0}^{|t|} \phi^{-1}(s) d s$, the complementary function of $\Phi$. (See [3] or [4] for the details of Young's function and its complement.) Our first result is

Theorem 1. Let $w, v$ be nonnegative, locally integrable functions on $R^{+}$, and suppose that $\Phi$ is a Young's function such that both $\Phi$ and its Young's complement $\Psi$ satisfy the $\Delta_{2}$-condition, i.e.,

$$
\Phi(2 t) \leq A \Phi(t), \quad \Psi(2 t) \leq B \Psi(t), \quad \text { for all } t \in R^{+} .
$$

Then, with $T$ defined in (1.2), there exists a constant $C$, independent of $f$, such that

$$
\int_{0}^{\infty} \Phi(T f(x)) w(x) d x \leq C \int_{0}^{\infty} \Phi(f(x)) v(x) d x
$$

if and only if there exists a constant $K$ such that

$$
\left(\int_{x}^{\infty} \varepsilon w(t) d t\right) \phi\left(\int_{0}^{x} \phi^{-1}\left(\frac{1}{\varepsilon v(t)}\right) d t\right) \leq K
$$

for all $\varepsilon>0$ and all $x>0$.

Furthermore, if we denote the best constants in (1.4) and (1.5) by $C$ and $K$, respectively, then there are positive constants $C_{1}, C_{2}, 0<\lambda_{1} \leq 1 \leq \lambda_{2} \leq$ $\lambda_{3}<\infty$, and $0<\theta \leq 1$ depending only on $\Phi$ such that

$$
C_{1} K \leq C \leq \begin{cases}C_{2} K^{\lambda_{1}} & \text { when } K \geq 1, \\ C_{2} K^{\lambda_{2}} & \text { when } \theta \leq K<1, \\ C_{2} K^{\lambda_{3}} & \text { when } 0<K<\theta .\end{cases}
$$

The special case $\Phi(t)=|t|^{p} \quad(1<p<\infty)$ of Theorem 1 is the well-known result of Muckenhoupt [5], which generalized the classical Hardy inequality

$$
\int_{0}^{\infty}\left(\frac{1}{x} \int_{0}^{x} f(t) d t\right)^{p} d x \leq\left(\frac{p}{p-1}\right)^{p} \int_{0}^{\infty} f(x)^{p} d x \text { for } f(x) \geq 0 \text { and } p>1 .
$$

Muckenhoupt's theorem is that given $p>1$ and a couple of nonnegative locally integrable weight functions $w(x)$ and $v(x)$, the inequality

$$
\left(\int_{0}^{\infty}\left|\int_{0}^{x} f(t) d t\right|^{p} w(x) d x\right)^{1 / p} \leq C\left(\int_{0}^{\infty}|f(x)|^{p} v(x) d x\right)^{1 / p}
$$

holds if and only if

$$
\sup _{r>0}\left(\int_{r}^{\infty} w(x) d x\right)^{1 / p}\left(\int_{0}^{r} v(x)^{-1 /(p-1)} d x\right)^{1 / p^{\prime}}=K<\infty
$$

and $K \leq C \leq p^{1 / p^{\prime}}\left(p^{\prime}\right)^{2 / p^{\prime}} K$, where $1 / p+1 / p^{\prime}=1$. In these cases the constants $K$ appearing in (1.6) and in Muckenhoupt's theorem are equivalent.

Our second theorem concerns fractional integrals in $R^{n}$. Following Sawyer in [8], we work with general convolution operators of the form

$$
T(f \mu)(x)=K^{*}(f \mu)(x)=\int_{R^{n}} K(x-y) f(y) d \mu(y),
$$


where the convolution kernel $K(x)$ is a positive lower semicontinuous radial function decreasing in $|x|$ and satisfying the growth condition $K(x) \leq C K(2 x)$ for every $x \in R^{n}$. We shall write $\chi_{E}$ for the characteristic function of a set $E \subset R^{n}$, and put $|E|_{\mu}=\int_{E} d \mu$. The Luxemberg norm $\|\cdot\|_{\Phi(\mu)}$ on the Orlicz space $L_{\Phi(\mu)}=\left\{f: \int_{R^{n}} \Phi(|f(x)|) d \mu(x)<\infty\right\}$ is given by

$$
\|f\|_{\Phi(\mu)}=\inf \left\{\theta>0: \int_{R^{n}} \Phi\left(\frac{|f(x)|}{\theta}\right) d \mu(x) \leq 1\right\} .
$$

With these notations we have

Theorem 2. Let $\Phi$ be a Young's function as in Theorem 1, $T$ be as defined in (1.7), and $d w, d \mu$ be positive Borel measures on $R^{n}$. Then in order that there exists a constant $C$ independent of $f$ such that

$$
\int_{R^{n}} \boldsymbol{\Phi}(T(f \mu)(x)) d w(x) \leq C \int_{R^{n}} \Phi(f(x)) d \mu(x)
$$

for all $f \geq 0$, it is necessary and sufficient that there is a constant $C$ such that both

$$
\int_{R^{n}} \Phi\left(T\left(\varepsilon \chi_{Q} \mu\right)(x)\right) d w(x) \leq C \Phi(\varepsilon)|Q|_{\mu}<\infty
$$

and

$$
\left\|T\left(\chi_{Q} w\right)\right\|_{\Psi(\varepsilon \mu)} \leq C\left\|\chi_{Q}\right\|_{\Psi(\varepsilon w)}<\infty
$$

hold for all $\varepsilon>0$ and all dyadic cubes $Q$.

In $[7,8]$ Sawyer and Wheeden discussed fractional integrals on weighted $L^{p}$ spaces. Our Theorem 2 is a generalization of Sawyer's result in [8].

Finally, we discuss the celebrated Hardy-Littlewood maximal function

$$
M f(x)=\sup _{x \in Q, \text { cube }} \frac{1}{|Q|} \int_{Q}|f(y)| d y .
$$

In fact, we shall deal with the following variant of the maximal function due to Fefferman and Stein (see [1]):

$$
T f(x, t)=M f(x, t)=\sup _{Q} \frac{1}{|Q|} \int_{Q}|f(y)| d y, \quad x \in R^{n}, t \geq 0,
$$

where the supremum is taken over all the cubes $Q$ in $R^{n}$, containing $x$ and having side length $l(Q) \geq t$. For this operator we obtain

Theorem 3. Suppose $d \mu$ is a positive Borel measure on $R_{+}^{n+1}=\{(x, t): x \in$ $\left.R^{n}, t \geq 0\right\}$ and $w(x)$ is a nonnegative locally integrable weight function on $R^{n}$. Let $\Phi$ be a Young's function that satisfies the $\Delta^{\prime}$-condition (see [4])

$$
\Phi(u v) \leq C \Phi(u) \Phi(v) \text { for all } u, v>0
$$

and its complement $\Psi$ still obey the $\Delta_{2}$-condition. Then, for $T$ defined in (1.13), in order that there exists a constant $C$ independent of $f$ such that

$$
\int_{R_{+}^{n+1}} \Phi(T(f w)(x, t)) d \mu(x, t) \leq C \int_{R^{n}} \Phi(f(x)) w(x) d x
$$


it is necessary and sufficient that there is a constant $C$ such that

$$
\int_{\widehat{Q}} \Phi\left(T\left(w \chi_{Q}\right)(x, t)\right) d \mu(x, t) \leq C|Q|_{w}<\infty
$$

for all cubes $Q$, where $\widehat{Q}=\{(x, t): x \in Q, 0 \leq t \leq l(Q)\}$.

It is well known that the two-weight norm inequality for the Hardy maximal operator defined in (1.12) has been characterized by Sawyer [9]. Later on, a similar result for the Fefferman-Stein maximal function was obtained in [6]. Our Theorem 3 extends their results.

In this paper we need the following elementary properties of Young's function $\Phi$ and its complement $\Psi$ (see [4] or [3]):

$$
t<\Phi^{-1}(t) \Psi^{-1}(t) \leq 2 t
$$

and

$$
\Phi(t) \sim t \phi(t), \quad \Psi(t) \sim t \phi^{-1}(t),
$$

when $\Phi$ and $\Psi$ both satisfy the $\Delta_{2}$-condition. The symbol ' $\sim$ ' means the ratio of the two sides is bounded between absolute positive constants.

\section{Proof of THEOREMS}

Proof of Theorem 1. Necessity. If $f \geq 0$ and $\operatorname{supp} f \subset[0, x]$ for given $x$, then from (1.4) it follows that

$$
\Phi\left(\int_{0}^{x} f(t) d t\right) \int_{x}^{\infty} w(t) d t \leq \int_{0}^{\infty} \Phi(T f(t)) w(t) d t \leq C \int_{0}^{x} \Phi(f(t)) v(t) d t
$$

Set $f(t)=\phi^{-1}(1 / \varepsilon v(t)) \chi_{[0, x]}(t)$. If $\int_{0}^{x} f(t) d t=0$, we have

$$
\left(\int_{x}^{\infty} \varepsilon w\right) \phi\left(\int_{0}^{x} \phi^{-1}(1 / \varepsilon v)\right)=0 \text {. }
$$

If $\int_{0}^{x} f(t) d t=\infty$, then $\int_{0}^{x} \Psi(1 / \varepsilon v(t)) \varepsilon v(t) d t=\infty$ because of (1.18). This implies that there is a nonnegative $g(t)$ such that $\int \Phi(g) \varepsilon v<\infty$ and $\int_{0}^{x} g(t)(1 / \varepsilon v(t)) \varepsilon v(t) d t=\infty$. Thus inequality (1.4) ensures that for arbitrary $\lambda>0$,

$$
\begin{aligned}
|[x, \infty]|_{\varepsilon w} & \leq|\{s: T g(s)>\lambda\}|_{\varepsilon w}=|\{s: \Phi(T g(s))>\Phi(\lambda)\}|_{\varepsilon w} \\
& \leq \frac{1}{\Phi(\lambda)} \int_{0}^{\infty} \Phi(T g) \varepsilon w \leq \frac{C}{\Phi(\lambda)} \int_{0}^{\infty} \Phi(g) \varepsilon v=\frac{C}{\Phi(\lambda)}
\end{aligned}
$$

Therefore $|[x, \infty]|_{\varepsilon w}=0$. Finally, we consider the case $0<\int_{0}^{x} f(t) d t<\infty$. Using (1.18) and (2.1), we obtain

$$
\begin{gathered}
\left(\int_{0}^{x} \phi^{-1}\left(\frac{1}{\varepsilon v}\right)\right) \phi\left(\int_{0}^{x} \phi^{-1}\left(\frac{1}{\varepsilon v}\right)\right) \int_{x}^{\infty} w \leq C \int_{x}^{\infty} \Phi(T f) w \\
\leq C \int_{0}^{\infty} \Phi(f) v \leq C \int_{0}^{x} \phi^{-1}\left(\frac{1}{\varepsilon v}\right) \phi\left(\phi^{-1}\left(\frac{1}{\varepsilon v}\right)\right) v
\end{gathered}
$$

This is (1.5). 
Sufficiency. We need the following inequality: We will show that condition (1.5) implies that

$$
\left\|\frac{\chi_{[0, x]}}{\varepsilon v}\right\|_{\Psi(\varepsilon v)} \leq C \Phi^{-1}\left(\frac{1}{\varepsilon \int_{x}^{\infty} w}\right)
$$

holds for all $\varepsilon>0$ and all $x>0$.

Indeed, from (1.5) it follows that

$$
\frac{\int_{0}^{x} \phi^{-1}(1 / \varepsilon v)}{\phi^{-1}\left(1 / \varepsilon \int_{x}^{\infty} w\right)} \leq C
$$

Setting $\eta=\eta_{x, \varepsilon}$ such that $\eta \varepsilon \Psi\left(1 / \varepsilon \int_{x}^{\infty} w\right) \int_{x}^{\infty} w=1$, we have

$$
\frac{1}{\eta} \sim \phi^{-1}\left(\frac{1}{\varepsilon \int_{x}^{\infty} w}\right) \sim \Phi^{-1}\left(\frac{1}{\eta \varepsilon \int_{x}^{\infty} w}\right)
$$

by use of (1.17) and (1.18). Substituting (2.5) into (2.4) and using (1.18), we obtain

$$
\int_{0}^{x} \Psi\left(\frac{1}{\varepsilon v(t)}\right) \eta \varepsilon v(t) d t \leq C,
$$

thus

$$
\left\|\frac{\chi_{[0, x]}}{\varepsilon \eta v}\right\|_{\Psi(\eta \varepsilon v)} \leq \frac{C}{\eta} \leq C \Phi^{-1}\left(\frac{1}{\eta \varepsilon \int_{x}^{\infty} w}\right) .
$$

Since $\eta \varepsilon=1 /\left(\Psi\left(1 / \varepsilon \int_{x}^{\infty} w\right) \int_{x}^{\infty} w\right)$ is a continuous function of $\varepsilon$ and takes values from 0 to $\infty$, we can have $\eta \varepsilon=\delta$ for $\delta$ given arbitrarily, and we conclude (2.3).

Now we can assume $f \geq 0$ and choose $x_{k}$ such that $T f\left(x_{k}\right)=\int_{0}^{x_{k}} f(t) d t=$ $2^{k}$ for all integers $k$ if it is possible. Then we have

$$
\begin{aligned}
\int_{0}^{\infty} \Phi(T f) w & \leq \sum_{k} \Phi\left(2^{k+1}\right) \int_{x_{k}}^{x_{k+1}} w \leq C \sum_{k} \Phi\left(2^{k-1}\right) \int_{x_{k}}^{\infty} w \\
& =C \sum_{k} \Phi\left(\int_{x_{k-1}}^{x_{k}} f(t) d t\right) \int_{x_{k}}^{\infty} w .
\end{aligned}
$$

Let $k$ be fixed and set $f_{k}=f(t) \chi_{\left(x_{k-1}, x_{k}\right]}(t)$. The Hölder inequality in Orlicz spaces shows that for any $\varepsilon_{k}>0$ given,

$$
\int_{x_{k-1}}^{x_{k}} f \leq C\left\|f_{k}\right\|_{\boldsymbol{\Phi}\left(\varepsilon_{k} v\right)}\left\|\frac{\chi_{\left[0, x_{k}\right]}}{\varepsilon_{k} v}\right\|_{\Psi\left(\varepsilon_{k} v\right)} .
$$

Choose $\varepsilon_{k}$ such that $\int \Phi\left(f_{k}\right) \varepsilon_{k} v=1$. Then $\left\|f_{k}\right\|_{\Phi\left(\varepsilon_{k} v\right)} \leq 1$. Thus, from (2.3) it follows that

$$
\int_{x_{k-1}}^{x_{k}} f \leq C \Phi^{-1}\left(\frac{1}{\varepsilon_{k} \int_{x_{k}}^{\infty} w}\right)
$$

therefore,

$$
\Phi\left(\int_{x_{k-1}}^{x_{k}} f\right) \leq \frac{C}{\varepsilon_{k} \int_{x_{k}}^{\infty} w}=C \frac{\int \Phi\left(f_{k}\right) v}{\int_{x_{k}}^{\infty} w} .
$$


Substituting (2.7) into (2.6) and observing that the supports of the $f_{k}$ are disjoint, we have

$$
\int_{0}^{\infty} \Phi(T f) w \leq C \sum_{k} \int \Phi\left(f_{k}\right) v=C \int_{0}^{\infty} \Phi(f) v .
$$

To discuss the relation between the best constant $C$ in (1.4) and $K$ in (1.5), we introduce some indices concerning $\Phi$ and $\Psi$. Define

$$
\begin{aligned}
& q_{\Phi}=\inf _{u>0} \frac{u \phi(u)}{\Phi(u)} \leq \sup _{u>0} \frac{u \phi(u)}{\Phi(u)}=p_{\Phi}, \\
& p_{\Phi}^{\prime}=\inf _{u>0} \frac{u \phi^{-1}(u)}{\Psi(u)} \leq \sup _{u>0} \frac{u \phi^{-1}(u)}{\Psi(u)}=q_{\Phi}^{\prime} .
\end{aligned}
$$

Then $1 / p_{\Phi}+1 / p_{\Phi}^{\prime}=1$ and $1 / q_{\Phi}+1 / q_{\Phi}^{\prime}=1$. Since both $\Phi$ and $\Psi$ satisfy the $\Delta_{2}$-condition, we have $1<q_{\Phi}, p_{\Phi}<\infty$ (see [4]). Thus equalities (2.8) imply that

$$
\begin{array}{ll}
\lambda^{q_{\Phi}} \Phi(u) \leq \Phi(\lambda u) \leq \lambda^{p_{\Phi}} \Phi(u) & \text { when } \lambda \geq 1, \\
\lambda^{p} \Phi(u) \leq \Phi(\lambda u) \leq \lambda^{q_{\Phi}} \Phi(u) & \text { when } \lambda<1
\end{array}
$$

hold for any $u>0$. Also analogous inequalities hold for $\Psi$. By use of these inequalities, (1.17), and (1.18), it is easy to obtain the estimate (1.6). For example, $C_{1}=q_{\Phi} / p_{\Phi}$ follows from the derivation of (2.2) since $q_{\Phi} \leq u \phi(u) / \Phi(u) \leq p_{\Phi}$. Moreover, the three power indices in (1.6) are $\lambda_{i}=1 \quad(i=1,2,3)$ when $\Phi(u)=|u|^{p}$. This completes the proof of Theorem 1 .

Proof of Theorem 2. If (1.9) holds, it is obvious that the dual norm inequality

$$
\|T(g w)\|_{\Psi(\varepsilon \mu)} \leq C\|g\|_{\Psi(\varepsilon w)}
$$

is true for all $\varepsilon>0$ and all $g \geq 0$. With $f=\varepsilon \chi_{Q}$ in (1.9) and $g=\chi_{Q}$ in (2.10), we obtain (1.10) and (1.11).

Conversely, suppose (1.10) and (1.11) hold and, without loss of generality, that $f$ is nonnegative and bounded with compact support. For every integer $k$ we consider the open set $\Omega_{k}=\left\{T(f \mu)>2^{k}\right\}$. From the Whitney decomposition lemma (cf. [8]), we obtain a sequence of dyadic cubes $\left\{Q_{j}^{k}\right\}$ satisfying

(1) $\Omega_{k}=\bigcup_{j} Q_{j}^{k}$ and $\left(Q_{j}^{k}\right)^{0} \cap\left(Q_{i}^{k}\right)^{0}=\varnothing$ for $i \neq j$;

(2) $R Q_{j}^{k} \subset \Omega_{k}$ and $3 R Q_{j}^{k} \cap \Omega_{k}^{c} \neq \varnothing$ for all $k, j$;

(3) $\sum_{j} \chi_{3 Q_{j}^{k}} \leq C \chi_{\Omega_{k}}$ for all $k$;

(4) the number of cubes $Q_{s}^{k}$ intersecting a fixed cube $3 Q_{j}^{k}$ is at most $C$;

(5) $Q_{j}^{k} \varsubsetneqq Q_{i}^{s}$ implies $k>s$.

In (2.11), $E^{0}$ is the interior of set $E$, and $R Q$ denotes the cube concentric with $Q$ with $R$ times the side length, the constant $R$ in (2.11)(2) being $\geq 3$ and depending only on the dimension $n$.

As in [8], we choose an integer $m \geq 2$ sufficiently large, depending only on the growth condition of $K(x)$ defined in (1.7). Write $E_{j}^{k}=Q_{j}^{k} \cap\left(\Omega_{k+m-1} \backslash \Omega_{k+m}\right)$ 
for all $(k, j)$. It is established in [8] that

$$
\begin{aligned}
\left|E_{j}^{k}\right|_{w} & \leq 2^{-k}\left(\int_{3 Q_{j}^{k} \backslash \Omega_{k+m}} f T\left(\chi_{E_{j}^{k}} w\right) d \mu+\int_{3 Q_{j}^{k} \cap \Omega_{k+m}} f T\left(\chi_{E_{j}^{k}} w\right) d \mu\right) \\
& =2^{-k}\left(\sigma_{j}^{k}+r_{j}^{k}\right) .
\end{aligned}
$$

Let $\beta \in(0,1)$, to be chosen later, and define

$$
\begin{aligned}
& E=\left\{(k, j):\left|E_{j}^{k}\right|_{w} \leq \beta\left|Q_{j}^{k}\right|_{w}\right\}, \\
& F=\left\{(k, j):\left|E_{j}^{k}\right|_{w}>\beta\left|Q_{j}^{k}\right|_{w} \text { and } \sigma_{j}^{k}>r_{j}^{k}\right\}, \\
& G=\left\{(k, j):\left|E_{j}^{k}\right|_{w}>\beta\left|Q_{j}^{k}\right|_{w} \text { and } \sigma_{j}^{k} \leq r_{j}^{k}\right\} .
\end{aligned}
$$

Thus we have

$$
\begin{aligned}
\int \Phi(T(f \mu)) d w & \leq \sum_{k} \Phi\left(2^{k+m}\right)\left|\Omega_{k+m-1} \backslash \Omega_{k+m}\right|_{w} \leq C \sum_{k, j}\left|E_{j}^{k}\right|_{w} \Phi\left(2^{k}\right) \\
& =C\left(\sum_{(k, j) \in E}+\sum_{(k, j) \in F}+\sum_{(k, j) \in G}\right)\left|E_{j}^{k}\right|_{w} \Phi\left(2^{k}\right) \\
& =C(\mathbf{I}+\mathbf{I I}+\mathbf{I I I}) .
\end{aligned}
$$

For the term I, we have

$$
\begin{aligned}
\mathrm{I} & \leq \sum_{k, j} \beta\left|Q_{j}^{k}\right|_{w} \Phi\left(2^{k}\right) \leq C \beta \sum_{k} \Phi\left(2^{k-1}\right)\left|\Omega_{k}\right|_{w} \\
& \leq C \beta \sum_{k} \int_{2^{k-1}}^{2^{k}}|\{T(f \mu)>\lambda\}|_{w} d \Phi(\lambda)=C \beta \int_{R^{n}} \Phi(T(f \mu)) d w .
\end{aligned}
$$

Now we estimate the term II:

$$
\begin{aligned}
\mathbf{I I} & \leq \sum_{(k, j) \in F}\left|E_{j}^{k}\right|_{w} \Phi\left(\frac{2 \sigma_{j}^{k}}{\left|E_{j}^{k}\right|_{w}}\right) \leq \sum\left|E_{j}^{k}\right|_{w} \Phi\left(\frac{2 \sigma_{j}^{k}}{\beta\left|E_{j}^{k}\right|_{w}}\right) \\
& \leq C_{\beta} \sum\left|E_{j}^{k}\right|_{w} \Phi\left(\frac{1}{\left|Q_{j}^{k}\right|_{w}} \int_{3 Q_{j}^{k} \backslash \Omega_{k+m}} f T\left(\chi_{E_{j}^{k}} w\right) d \mu\right),
\end{aligned}
$$

where $C_{\beta}=(2 / \beta)^{p_{\Phi}}$ by use of $(2.9)$. have

Let $(k, j)$ be fixed and let $\varepsilon=\varepsilon_{k, j}>0$. On writing $R_{j}^{k}=3 Q_{j}^{k} \backslash \Omega_{k+m}$, we

$$
\frac{1}{\left|Q_{j}^{k}\right|_{w}} \int_{3 Q_{j}^{k} \backslash \Omega_{k+m}} f T\left(\chi_{E_{j}^{k}} w\right) d \mu \leq \frac{1}{\varepsilon_{k, j}\left|Q_{j}^{k}\right|_{w}}\left\|f \chi_{R_{j}^{k}}\right\|_{\Phi(\varepsilon \mu)}\left\|T\left(\chi_{Q_{j}^{k}} w\right)\right\|_{\Psi(\varepsilon \mu)} .
$$

If $\varepsilon=\varepsilon_{k, j}$ is chosen such that $\int \Phi\left(f \chi_{R_{j}^{k}}\right) \varepsilon_{k, j} d \mu=1$ then $\left\|f \chi_{R_{j}^{k}}\right\|_{\Phi(\varepsilon \mu)} \leq 1$, and from (1.11) it follows that

$$
\left\|T\left(\chi_{Q_{j}^{k}} w\right)\right\|_{\Psi(\varepsilon \mu)} \leq C\left\|\chi_{Q_{j}^{k}}\right\|_{\Psi(\varepsilon w)} \leq C \Phi^{-1}\left(\frac{1}{\varepsilon_{k, j}\left|Q_{j}^{k}\right|_{w}}\right) \varepsilon_{k, j}\left|Q_{j}^{k}\right|_{w} .
$$


Therefore, we obtain

$$
\frac{1}{\left|Q_{j}^{k}\right|_{w}} \int_{R_{j}^{k}} f T\left(\chi_{E_{j}^{k}} w\right) d \mu \leq C \Phi^{-1}\left(\frac{1}{\varepsilon_{k, j}\left|Q_{j}^{k}\right|_{w}}\right) .
$$

On substituting the last estimate into (2.15), it follows that

$$
\begin{aligned}
\mathrm{II} & \leq C_{\beta} C \sum_{k, j} \frac{\left|E_{j}^{k}\right|_{w}}{\varepsilon_{k, j}\left|Q_{j}^{k}\right|_{w}} \leq C \sum_{k, j} \int \Phi\left(f \chi_{R_{j}^{k}}\right) d \mu \\
& \leq C \int_{R^{n}} \Phi(f) d \mu .
\end{aligned}
$$

The last inequality holds since $\sum_{k, j} \chi_{R_{j}^{k}} \leq C \sum_{k} \chi_{\Omega_{k} \backslash \Omega_{k+m}} \leq C(m+1)$ by $(2.11)(3)$.

To estimate the term III in (2.13), we need the following $\Phi$-inequality for the dyadic maximal operator. For $\sigma$ a positive Borel measure on $R^{n}$, define

$$
M_{\sigma}^{d} f(x)=\sup _{x \in Q, \text { dyadic cube }} \frac{1}{|Q|_{\sigma}} \int_{Q}|f(y)| d \sigma(y) \text { for } f \in L_{\mathrm{loc}}(\sigma) .
$$

Then for any given Young's function $\Phi$ as in Theorem 2,

$$
\int_{R^{n}} \Phi\left(M_{\sigma}^{d} f\right) d \sigma C \int_{R^{n}} \Phi(f) d \sigma
$$

holds for all $f \in L_{\Phi}(\sigma)$.

Indeed, it is known that the operator $M_{\sigma}^{d}$ is weak type $(1,1)$ with the constant 1 with respect to the measure $\sigma$. It follows that

$$
\begin{aligned}
\int_{R^{n}} \Phi\left(M_{\sigma}^{d} f\right) d \sigma & \leq C \int_{0}^{\infty}\left|\left\{M_{\sigma}^{d} f>2 \lambda\right\}\right|_{\sigma} \phi(\lambda) d \lambda \\
& \leq C \int_{0}^{\infty}\left(\frac{1}{\lambda} \int_{\{|f|>\lambda\}}|f| d \sigma\right) \frac{\Phi(\lambda)}{\lambda} d \lambda \\
& =C \int_{R^{n}} \int_{0}^{|f|} \frac{\Phi(\lambda)}{\lambda^{2}} d \lambda|f| d \sigma \leq C \int_{R^{n}} \Phi(|f|) d \sigma
\end{aligned}
$$

since

$$
\int_{0}^{u} \frac{\Phi(\lambda)}{\lambda^{2}} d \lambda=\frac{1}{u} \int_{0}^{1} \frac{\Phi(t u)}{t^{2}} d t \leq \frac{\Phi(u)}{u} \int_{0}^{1} \frac{d t}{t^{2-q_{\Phi}}}=C \frac{\Phi(u)}{u} .
$$

Let $H_{j}^{k}=\left\{i: Q_{i}^{k+m} \cap 3 Q_{j}^{k} \neq \varnothing\right\}$ and $L_{j}^{k}=\left\{s: Q_{s}^{k} \cap 3 Q_{j}^{k} \neq \varnothing\right\}$. Then for given $(k, j)$,

$$
3 Q_{j}^{k} \cap \Omega_{k+m} \subset \bigcup_{i \in H_{j}^{k}} Q_{i}^{k+m} \subset \bigcup_{s \in L_{j}^{k}} \bigcup_{i: Q_{i}^{k+m} \subset Q_{s}^{k}} Q_{i}^{k+m} .
$$

Set $A_{j}^{k}=\left(1 /\left|Q_{j}^{k}\right|_{\mu}\right) \int_{Q_{j}^{k}} f d \mu$. In [8] it has been obtained that

$$
r_{j}^{k} \leq C \sum_{i \in H_{j}^{k}}\left(\int_{Q_{i}^{k+m}} T\left(\chi_{E_{j}^{k}} w\right) d \mu\right) A_{i}^{k+m}
$$


Following the approach in [8], we shall prove that

$$
\sum_{\substack{(k, j) \in G \\ k \geq N, \bmod m)}}\left|E_{j}^{k}\right|_{w} \Phi\left(2^{k}\right) \leq C \int_{R^{n}} \Phi(f) d \mu
$$

with a constant $C$ independent of the integers $N$ and $M$, where $N \in(-\infty, \infty)$, $0 \leq M<m$. The indices $(k, j)$ are restricted by this convention until the proof of $(2.20)$ is completed.

As in [8], we select the "principle" cubes from $\left\{Q_{j}^{k}\right\}$. Let $G_{0}$ consist of those indices $(k, j)$ for which $Q_{j}^{k}$ is maximal. If $G_{n}$ has been defined, for every index $(t, u)$ in $G_{n}$ we select the maximal cubes $Q_{j}^{k} \subset Q_{u}^{t}$ such that $A_{j}^{k}>2 A_{u}^{t}$. The indices of those cubes so selected form the set $G_{n+1}$. Define $\Gamma=\bigcup_{n=0}^{\infty} G_{n}$, and for each $(k, j)$, define $P\left(Q_{j}^{k}\right)$ to be the smallest cube $Q_{u}^{t}$ containing $Q_{j}^{k}$ with $(t, u) \in \Gamma$. Then we have

(1) $P\left(Q_{j}^{k}\right)=Q_{u}^{t}$ implies $A_{j}^{k} \leq 2 A_{u}^{t}$;

(2) $Q_{j}^{k} \subset Q_{u}^{t}$ and $(k, j),(t, u) \in \Gamma$ imply $A_{j}^{k}>2 A_{u}^{t}$.

Observing that the cardinality of $L_{j}^{k}$ is at most $C$ and if $Q_{i}^{k+m} \subset Q_{s}^{k}$ with $(k+m, i) \notin \Gamma$ then $P\left(Q_{i}^{k+m}\right)=P\left(Q_{s}^{k}\right)$, we obtain

$$
\begin{aligned}
& \sum_{(k, j) \in G}\left|E_{j}^{k}\right|_{w} \Phi\left(2^{k}\right) \leq C \sum\left|E_{j}^{k}\right|_{w} \Phi\left(\frac{r_{j}^{k}}{\left|Q_{j}^{k}\right|_{w}}\right) \quad \text { (by (2.12) and (2.8)) } \\
& \leq C \sum\left|E_{j}^{k}\right|_{w} \Phi\left(\frac{1}{\left|Q_{j}^{k}\right|_{w}} \sum_{\substack{s \in L_{j}^{k} \\
i: P\left(Q_{i}^{k+m}\right)=P\left(Q_{s}^{k}\right), Q_{i}^{k+m} \subset Q_{s}^{k}}}\left[\left(\int_{Q_{i}^{k+m}} T\left(\chi_{Q_{j}^{k}} w\right) d \mu\right) A_{i}^{k+m}\right]\right) \\
& +C \sum\left|E_{j}^{k}\right|_{w} \Phi\left(\frac{1}{\left|Q_{j}^{k}\right|_{w}} \sum_{i \in H_{j}^{k}:(k+m, j) \in \Gamma}\left[\left(\int_{Q_{i}^{k+m}} T\left(\chi_{Q_{j}^{k}} w\right) d \mu\right) A_{i}^{k+m}\right]\right) \\
& \leq C \sum\left|E_{j}^{k}\right|_{w} \sum_{s \in L_{j}^{k}} \Phi\left(\frac{1}{\left|Q_{j}^{k}\right|_{w}} \sum_{\substack{i: P\left(Q_{i}^{k+m}\right)=P\left(Q_{s}^{k}\right), Q_{i}^{k+m} \subset Q_{s}^{k}}}\left[\left(\int_{Q_{i}^{k+m}} T\left(\chi_{Q_{j}^{k}} w\right) d \mu\right) A_{i}^{k+m}\right]\right) \\
& +C \sum\left|E_{j}^{k}\right|_{w} \Phi\left(\frac{1}{\left|Q_{j}^{k}\right|_{w}} \sum_{i \in H_{j}^{k}:(k+m, j) \in \Gamma}\left[\left(\int_{Q_{i}^{k+m}} T\left(\chi_{Q_{j}^{k}} w\right) d \mu\right) A_{i}^{k+m}\right]\right) \\
& =C(\mathrm{IV}+\mathrm{V}) \text {. }
\end{aligned}
$$


To deal with IV, we deduce the following estimate for fixed $(t, u) \in \Gamma$.

$$
\begin{aligned}
& \sum_{k, j} \sum_{s \in L_{j}^{k}: P\left(Q_{s}^{k}\right)=Q_{u}^{t}}\left|E_{j}^{k}\right|_{w} \Phi\left(\frac{1}{\left|Q_{j}^{k}\right|_{w}} \sum_{\substack{i: P\left(Q_{i}^{k+m}\right)=P\left(Q_{s}^{k}\right), Q_{i}^{k+m} C Q_{s}^{k}}}\left[\left(\int_{Q_{i}^{k+m}} T\left(\chi_{Q_{j}^{k}} w\right) d \mu\right) A_{i}^{k+m}\right]\right) \\
& \quad \leq \sum_{k, j} \sum_{s \in L_{j}^{k}: P\left(Q_{s}^{k}\right)=Q_{u}^{t}}\left|E_{j}^{k}\right|_{w} \Phi\left(\frac{2 A_{u}^{t}}{\left|Q_{j}^{k}\right|_{w}} \int_{Q_{s}^{k}} T\left(\chi_{Q_{j}^{k}} w\right) d \mu\right) \quad(\text { by }(2.21)(1)) \\
& \leq C \sum_{k, j} \sum_{s \in L_{j}^{k}: P\left(Q_{s}^{k}\right)=Q_{u}^{t}}\left|E_{j}^{k}\right|_{w} \Phi\left(\frac{1}{\left|Q_{j}^{k}\right|_{w}} \int_{Q_{j}^{k}} T\left(A_{u}^{t} \chi_{Q_{u}^{t}} \mu\right) d w\right) \\
& \leq C \sum_{k, j} \int_{E_{j}^{k}} \Phi\left(M_{w}^{d}\left(T\left(A_{u}^{t} \chi_{Q_{u}^{t}} \mu\right)\right)\right) d w \quad\left(\text { since the cardinality of } L_{j}^{k} \leq C\right) \\
& \leq C \int \Phi\left(T\left(A_{u}^{t} \chi_{Q_{u}^{t}} \mu\right)\right) d w \quad(\text { by }(2.18)) \\
& \leq C \Phi\left(A_{u}^{t}\right)\left|Q_{u}^{t}\right|_{\mu} \quad(\text { by }(1.10)) .
\end{aligned}
$$

Summing (2.23) over $(t, u) \in \Gamma$ yields

$$
\mathrm{IV} \leq C \sum_{(t, u) \in \Gamma} \Phi\left(A_{u}^{t}\right)\left|Q_{u}^{t}\right|_{\mu} .
$$

Now we estimate V. For every $(k, j)$ let $\Gamma_{j}^{k}=\left\{i: i \in H_{j}^{k},(k+m, i) \in \Gamma\right\}$ and $P_{j}^{k}=\bigcup_{i \in \Gamma_{j}^{k}} Q_{i}^{k+m}$. Then

$$
\begin{aligned}
V & =\sum_{(k, j) \in G}\left|E_{j}^{k}\right|_{w} \Phi\left(\frac{1}{\left|Q_{j}^{k}\right|_{w}} \sum_{i \in \Gamma_{j}^{k}}\left[\left(\int_{Q_{i}^{k+m}} T\left(\chi_{Q_{j}^{k}} w\right) d \mu\right) A_{i}^{k+m}\right]\right) \\
& =\sum_{(k, j) \in G}\left|E_{j}^{k}\right|_{w} \Phi\left(\frac{1}{\left|Q_{j}^{k}\right|_{w}} \int_{P_{j}^{k}} T\left(\chi_{Q_{j}^{k}} w\right)\left(\sum_{i \in \Gamma_{j}^{k}} A_{i}^{k+m} \chi_{Q_{i}^{k+m}}\right) d \mu\right) .
\end{aligned}
$$

Let $(k, j)$ be fixed and let $\varepsilon=\varepsilon_{k, j}>0$. Using the Hölder inequality in the Orlicz spaces, we have

$$
\begin{aligned}
& \frac{1}{\left|Q_{j}^{k}\right|_{w}} \int_{P_{j}^{k}} T\left(\chi_{Q_{j}^{k}} w\right)\left(\sum_{i \in \Gamma_{j}^{k}} A_{i}^{k+m} \chi_{Q_{i}^{k+m}}\right) d \mu \\
& \quad \leq \frac{C}{\varepsilon\left|Q_{j}^{k}\right|_{w}}\left\|T\left(\chi_{Q_{j}^{k}} w\right)\right\| \Psi(\varepsilon \mu)\left\|\sum_{i \in \Gamma_{j}^{k}} A_{i}^{k+m} \chi_{Q_{i}^{k+m}}\right\|_{\Phi(\varepsilon \mu)} .
\end{aligned}
$$


If $\varepsilon_{k, j}$ is chosen such that $\int \Phi\left(\sum_{i \in \Gamma_{j}^{k}} A_{i}^{k+m} \chi_{Q_{i}^{k+m}}\right) \varepsilon_{k, j} d \mu=1$, then

$$
\left\|\sum_{i \in \Gamma_{j}^{k}} A_{i}^{k+m} \chi_{Q_{i}^{k+m}}\right\|_{\Phi(\varepsilon \mu)} \leq 1
$$

and by use of (2.16) and (2.26) we have

$$
\Phi\left(\frac{1}{\left|Q_{j}^{k}\right|_{w}} \int_{P_{j}^{k}} T\left(\chi_{Q_{j}^{k}} w\right)\left(\sum_{i \in \Gamma_{j}^{k}} A_{i}^{k+m} \chi_{Q_{i}^{k+m}}\right) d \mu\right) \leq \frac{C}{\varepsilon_{k, j}\left|Q_{j}^{k}\right|_{w}} .
$$

Therefore it follows that

$$
\begin{aligned}
V & \leq C \sum_{(k, j) \in G} \frac{\left|E_{j}^{k}\right|_{w}}{\varepsilon_{k, j}\left|Q_{j}^{k}\right|_{w}} \leq C \sum_{(k, j) \in G} \frac{1}{\varepsilon_{k, j}} \\
& =C \sum_{(k, j) \in G} \sum_{i \in \Gamma_{j}^{k}} \Phi\left(A_{i}^{k+m}\right)\left|Q_{i}^{k+m}\right|_{\mu} \leq C \sum_{(t, u) \in \Gamma} \Phi\left(A_{u}^{t}\right)\left|Q_{u}^{t}\right|_{\mu}
\end{aligned}
$$

since any fixed $Q_{i}^{k+m}$ occurs at most $C$ times in the above sum (see [7, 8]).

From (2.9) and (2.21)(2) it follows that for any fixed $x$

$$
\sum_{(t, u) \in \Gamma} \Phi\left(A_{u}^{t}\right) \chi_{Q_{u}^{t}}(x) \leq C \sup _{x \in Q_{u}^{t}} \Phi\left(A_{u}^{t}\right) \sum_{k=0}^{\infty} \frac{1}{2^{k q_{\Phi}}} \leq C \Phi\left(M_{\mu}^{d} f(x)\right) .
$$

Combining (2.22), (2.24), and (2.27) shows that the left side of (2.20) is bounded by

$$
\begin{aligned}
& C \sum_{(t, u) \in \Gamma} \Phi\left(A_{u}^{t}\right)\left|Q_{u}^{t}\right|_{\mu} \leq C \int \sum_{(t, u) \in \Gamma} \Phi\left(A_{u}^{t}\right) \chi_{Q_{u}^{t}}(x) d \mu \\
& \quad \leq C \int \Phi\left(M_{\mu}^{d} f(x)\right) d \mu \leq C \int \Phi(f) d \mu \quad \text { (by (2.18)). }
\end{aligned}
$$

Let $N \rightarrow \infty$ in (2.20) and then sum over $M=0,1, \ldots, m-1$ to obtain

$$
\mathrm{III} \leq C \int \Phi(f) d \mu .
$$

In (2.14) choose $\beta$ so small that $C \beta<\frac{1}{2}$. It is easy to conclude (1.9) from (2.13), (2.14), (2.17), and (2.29). Hence for arbitrary $f \geq 0$ we obtain (1.9) by the monotone convergence theorem. This completes the proof of Theorem 2.

Proof of Theorem 3. The proof of the necessity is easy and we omit it. For the sufficiency, first, we prove the theorem for the relative dyadic maximal operator

$$
N f(x, t)=\sup \frac{1}{|Q|} \int_{Q}|f(y)| d y, \quad x \in R^{n}, t \geq 0,
$$

where the supremum is taken over the dyadic cubes in $R^{n}$ containing $x$ and having side length at least $t$. In addition, assuming the side length at most $R$, we denote the associated operator by $N^{R}$. Obviously, $N^{R} f(x, t)=0$ for $t>R$ and $\lim N^{R} f(x, t)=N f(x, t) \quad(R \rightarrow \infty)$. 
We shall prove that (1.15) is true for $N^{R}$ with constant $C$ independent of $R$ if (1.16) holds for all dyadic cubes. Once this is proved, a limit argument shows that (1.15) holds for $N$.

Suppose $f \geq 0$. For every integer $k$ let $\Omega_{k}=\left\{(x, t): N^{R}(f w)>2^{k}\right\}$. According to a decomposition lemma in [6], there exists a family $\left\{Q_{j}^{k}\right\}_{j \in J_{k}}$ of dyadic cubes such that

(1) $\left(1 /\left|Q_{j}^{k}\right|\right) \int_{Q_{j}^{k}} f w>2^{k}$;

(2) the interiors of $Q_{j}^{k}$ are disjoint;

(3) $\Omega_{k}=\bigcup_{j \in J_{k}} \widehat{Q}_{j}^{k}$.

Writing $E_{j}^{k}=\widehat{Q}_{j}^{k} \backslash \Omega_{k+1}$ we have

$$
\begin{aligned}
\int_{R_{+}^{n+1}} \Phi\left(N^{R}(f w)\right) d \mu & \leq C \sum_{k, j} \Phi\left(2^{k}\right) \mu\left(E_{j}^{k}\right) \\
& \leq C \sum \mu\left(E_{j}^{k}\right) \Phi\left(\frac{\left|Q_{j}^{k}\right|_{w}}{\left|Q_{j}^{k}\right|}\right) \Phi\left(\frac{1}{\left|Q_{j}^{k}\right|_{w}} \int_{Q_{j}^{k}} f w\right)
\end{aligned}
$$

by use of $(2.30)(1)$ and the $\Delta^{\prime}$-condition. For every integer $s$ let

$$
\Gamma_{s}=\left\{(k, j): 2^{s}<\Phi\left(\frac{1}{\left|Q_{j}^{k}\right|_{w}} \int_{Q_{j}^{k}} f w\right) \leq 2^{s+1}\right\}, \quad G_{s}=\bigcup_{(k, j) \in \Gamma_{s}} Q_{j}^{k} .
$$

Since all the cubes in the doubly indexed family $\left\{Q_{j}^{k}:(k, j) \in \Gamma_{s}\right\}$ have side length $\leq R$, every cube will be contained in a maximal one. Let $\left\{Q_{i}\right\}_{i \in \Gamma_{s}^{\prime}}$ be the subfamily formed by these maximal cubes. Then $G_{s}=\bigcup_{i \in \Gamma_{s}^{\prime}} Q_{i} \subset\{x \in$ $\left.R^{n}: \Phi\left(M_{w}^{d} f(x)\right)>2^{s}\right\}$. Therefore the right side of (2.31) is bounded by

$$
\begin{aligned}
C \sum_{s=-\infty}^{\infty} & \sum_{i \in \Gamma_{s}^{\prime}(k, j) \in \Gamma_{s},} \sum_{\substack{Q_{j}^{k} \subset Q_{i}\\
}} \mu\left(E_{j}^{k}\right) \Phi\left(\frac{\left|Q_{j}^{k}\right|_{w}}{\left|Q_{j}^{k}\right|}\right) \Phi\left(\frac{1}{\left|Q_{j}^{k}\right|_{w}} \int_{Q_{j}^{k}} f w\right) \\
& \leq C \sum_{s=-\infty}^{\infty} \sum_{i \in \Gamma_{s}^{\prime}(k, j) \in \Gamma_{s},} \sum_{Q_{j}^{k} \subset Q_{i}} 2^{s+1} \int_{E_{j}^{k}} \Phi\left(N\left(\chi_{Q_{j}^{k}} w\right)(x, t)\right) d \mu(x, t) \\
& \leq C \sum_{s} 2^{s+1} \sum_{i \in \Gamma_{s}^{\prime}} \int_{\widehat{Q}_{i}} \Phi\left(N\left(\chi_{Q_{i}} w\right)(x, t)\right) d \mu(x, t) \\
& \leq C \sum_{s} 2^{s+1} \sum_{i \in \Gamma_{s}^{\prime}}\left|Q_{i}\right|_{w} \quad(\text { by }(1.16)) \\
& \leq C \sum_{s} 2^{s+1} \mid\left\{x \in R^{n}:\left.\Phi\left(M_{w}^{d}(f(x))>2^{s}\right\}\right|_{w}\right. \\
& \leq C \int \Phi\left(M_{w}^{d} f\right) w d x \leq C \int \Phi(f) w d x \quad \text { (by (2.18)). }
\end{aligned}
$$


The rest of the proof follows easily from the following variation of Sawyer's lemma that appeared in [6].

Lemma. Define for each $y \in R^{n}$

$$
{ }^{y} N f(x, t)=\sup \frac{1}{|Q|} \int_{Q}|f(u)| d u,
$$

the supremum being taken in all cubes $Q$ with $x \in Q$, side length more that $t$, and such that the set $Q-y=\{x-y: x \in Q\}$ is a dyadic cube. Then

$$
M^{2^{k}} f(x, t) \leq \frac{2^{3 n+1}}{\left|Q\left(0,2^{k+2}\right)\right|} \int_{Q\left(0,2^{k+2}\right)}{ }^{y} N f(x, t) d y,
$$

where $Q\left(0,2^{k+2}\right)=\left[-2^{k+2}, 2^{k+2}\right]^{n}$ and by $M^{r}$ we mean the maximal operator obtained by considering cubes with side length less than $r$. Then we have

$$
\begin{aligned}
& \int_{R_{+}^{n+1}} \Phi\left(M^{2^{k}}(f w)(x, t)\right) d \mu(x, t) \\
& \leq C \int_{R_{+}^{n+1}} \Phi\left(\frac{2^{3 n+1}}{\left|Q\left(0,2^{k+2}\right)\right|} \int_{Q\left(0,2^{k+2}\right)}{ }^{y} N f(x, t) d y\right) d \mu \\
& \leq \frac{C}{\left|Q\left(0,2^{k+2}\right)\right|} \int_{Q\left(0,2^{k+2}\right)} \int_{R_{+}^{n+1}} \Phi\left({ }^{y} N(f w)(x, t)\right) d \mu(x, t) d y \\
& \leq C \int \Phi(f) w d x
\end{aligned}
$$

By letting $k \rightarrow \infty$ we conclude (1.15). Theorem 3 is proved.

\section{ACKNOWLEDGMENT}

This article was finished during my visit to the University of Wales College of Cardiff. I wish to thank Professor W. D. Evans for many helpful suggestions and conversations.

\section{REFERENCES}

1. C. Fefferman and E. M. Stein, Some maximal inequalities, Amer. J. Math. 93 (1971), 107-115.

2. J. Carcia-Cuerva and J. L. Rubio De Francia, Weighted norm inequalities and related topics, North-Holland, Amsterdam, 1985.

3. R. A. Kerman and A. Torchinsky, Integral inequalities with weight for Hardy maximal function, Studia Math. 71 (1982), 277-284.

4. M. A. Krasnosel'skiĩ and Ya. B. Rutickiī, Convex functions and Orlicz spaces, Noordhoff, Gronigen, 1961.

5. B. Muckenhoupt, Hardy inequality with weights, Studia Math. 44 (1972), 31-38.

6. F. J. Ruiz and J. L. Torrea, A unified approach to Carleson measure and $A_{p}$ weights. II, Pacific J. Math. 120 (1985), 189-197.

7. E. T. Sawyer and R. L. Wheeden, Weighted inequalities for fractional integrals on Euclidean and homogeneous spaces (to appear). 
8. E. T. Sawyer, A characterization of two weight norm inequalities for fractional and Poisson integrals, Trans. Amer. Math. Soc. 308 (1988), 533-545.

9. _ A characterization of a two weight norm inequality for maximal operators, Studia Math. 75 (1982), 1-11.

Department of Mathematics and Mechanics, Central South University of Technology, Changsha Hunan, People's Republic of China

Current address: Department of Pure Mathematics, University of Leeds, Leeds, LS2 9JT, United Kingdom

E-mail address: PMT5LQ@UK.AC.LEEDS.CMS1 